\title{
Mesothelin Expression in Gastric Adenocarcinoma and Its Relation to Clinical Outcomes
}

\author{
Song-Hee Han · Mee Joo \\ Hanseong Kim · Sunhee Chang \\ Department of Pathology, Inje University Ilsan \\ Paik Hospital, Goyang, Korea
}

\begin{abstract}
Background: Although surgical resection with chemotherapy is considered effective for patients with advanced gastric cancer, it remains the third leading cause of cancer-related death in South Korea. Several studies have reported that mesothelial markers including mesothelin, calretinin, and Wilms tumor protein 1 (WT1) were positive in variable carcinomas, associated with prognosis, and were evaluated as potential markers for targeted therapy. The aim of this study was to assess the immunohistochemical expression of mesothelial markers (mesothelin, calretinin, and WT1) in gastric adenocarcinoma and their relations to clinocopathological features and prognosis. Methods: We evaluated calretinin, WT1, and mesothelin expression by immunohistochemical staining in 117 gastric adenocarcinomas. Results: Mesothelin was positively stained in 30 cases (25.6\%). Mesothelin expression was related to increased depth of invasion $(p=.002)$, lymph node metastasis $(p=.013)$, and presence of lymphovascular $(p=.015)$ and perineural invasion $(p=.004)$. Patients with mesothelin expression had significantly worse disease-free survival rate compared with that of nonmesothelin expression group $(p=.024)$. Univariate analysis showed that mesothelin expression is related to short-term survival. None of the 117 gastric adenocarcinomas stained for calretinin or WT1. Conclusions: Mesothelin expression was associated with poor prognosis. Our results suggest that mesothelin-targeted therapy should be considered as an important therapeutic alternative for gastric adenocarcinoma patients with mesothelin expression.
\end{abstract}

Key Words: Mesothelin; Gastric adenocarcinoma; Prognosis
Gastric cancer is a major health problem worldwide, causing approximately one million deaths every year. ${ }^{1}$ In South Korea, gastric cancer is the third leading cause of cancer-related death. ${ }^{2}$ With improved screening methods such as endoscopy and the development of new techniques such as endoscopic submucosal dissection, patients can be diagnosed with gastric cancer at an early stage and are expected to have increased long-term survival rate and higher quality of life. ${ }^{3}$ Although surgical resection with chemotherapy is considered effective for patients with recurrent, metastatic, or advanced gastric cancer (AGC), the 5-year survival rate for patients with AGC is only $20 \%-30 \% .{ }^{4}$ Chemotherapy increases survival rate, enhances quality of life, and can achieve symptomatic control; however, it is associated with increased drug toxicity. ${ }^{5}$ In recent years, the identification of druggable oncogenic alterations such as human epidermal growth factor receptor 2 (HER2) overexpression and the development of drugs that specifically target HER2 have led to substantial improvement in the prognosis of patients with AGC. ${ }^{6}$ However, oncogenic alterations are detected in only $6.0 \%-29.5 \%$ of gastric adenocarcinomas. ${ }^{7}$ Thus, development of novel effective targeted therapy is essential for the management of AGC.

Mesothelin, calretinin, and Wilms tumor protein 1 (WT1) are immunohistochemical markers for mesothelial differentiation. These markers are positive in various carcinomas and have been associated with prognosis. Mesothelin has been found to be expressed in some carcinomas, particularly those arising in the ovary, pancreas, and stomach, and has prognostic value. ${ }^{8-12} \mathrm{Cal}-$ retinin expression has been reported in carcinomas of various tissues including the ovary, testis, adrenal cortex, colon, breast, sinonasal tract, thymus, skin, and even soft tissues. ${ }^{13}$ Previous studies have reported the prognostic implications of calretinin expression in breast cancer and malignant mesothelioma. ${ }^{14,15} \mathrm{Qi}$ et al. ${ }^{16}$ reported that WT1-positive expression was associated with unfavorable clinical outcomes in patients with ovarian cancer, endometrial cancer, and noncarcinoma malignancies.

A few studies have investigated the expression of these mesothelial markers in gastric adenocarcinoma. Mesothelin expression percentages ranged from $15.4 \%$ to $59.4 \%$; however, conflicting results were reported on the relationship between prognosis of patients with gastric adenocarcinoma and positive 
mesothelin expression. ${ }^{11,12}$ Several studies have examined the importance of WT1 and calretinin expression in gastric adenocarcinoma. However, mesothelial marker expression in gastric adenocarcinoma and the prognostic value of such expression remain unclear.

To investigate the prognostic significance of mesothelial markers in gastric adenocarcinoma, we evaluated the expression of mesothelial markers (mesothelin, calretinin, and WT1) in gastric adenocarcinoma and the association between positive mesothelial markers and clinicopathological variables and disease-free survival (DFS).

\section{MATERIALS AND METHODS}

\section{Patients and tissue samples}

Subjects in this study totaled 117 patients who had histologically confirmed gastric adenocarcinoma and who underwent radical resection at Inje University Ilsan Hospital from January 2005 to December 2011. These specimens were composed of 86 early gastric cancer and 31 AGC cases. The patients enrolled in this study had not received neoadjuvant chemotherapy or radiation therapy prior to surgery. Patients were followed for
1-10 years. The clinicopathological features including patient age, gender, and other factors are shown in Table 1. Hematoxylin and eosin (H\&E)-stained slides for all cases were reviewed to confirm the diagnosis. The study was approved by the Institutional Review Board of Inje University Ilsan Hospital (ISPAIK 2015-10-017).

\section{Tissue microarray construction}

Paraffin blocks containing representative samples of the tumors were selected by reviewing all of the H\&E-stained slides. For the tissue microarray (TMA), two tissue cores with a diameter of $3 \mathrm{~mm}$ were extracted from a representative tumor area and were sequentially placed in recipient tissue array blocks using a tissue arraying instrument (Labro, Seoul, Korea). Sections from TMA blocks were prepared ( $4 \mu \mathrm{m}$ thickness) for routine H\&E staining. Other sections were prepared on charged slides for immunohistochemical staining.

\section{Immunohistochemical evaluation}

Immunohistochemistry was performed using standard methods. Formalin-fixed, $4 \mu \mathrm{m}$-thick, paraffin-embedded tissue sections were deparaffinized and subjected to antigen retrieval (im-

Table 1. Clinicopathological correlation of mesothelin expression in gastric adenocarcinoma

\begin{tabular}{|c|c|c|c|}
\hline Parameter & Mesothelin positive $(n=30,25.6 \%)$ & Mesothelin negative ( $n=87,74.4 \%)$ & $p$-value \\
\hline Age, mean (range, yr) & $66(41-89)$ & $66(35-91)$ & \\
\hline Gender & & & .110 \\
\hline Female & $8(20.0)$ & $32(80.0)$ & \\
\hline Male & $22(28.6)$ & $55(71.4)$ & \\
\hline Stage & & & .001 \\
\hline EGC & $16(18.6)$ & $70(81.4)$ & \\
\hline AGC & $14(45.2)$ & $17(54.8)$ & \\
\hline Lymph node metastasis & & & .013 \\
\hline Present & $16(38.1)$ & $26(61.9)$ & \\
\hline Absent & $14(18.7)$ & $61(81.3)$ & \\
\hline Lauren classification & & & .113 \\
\hline Intestinal & $17(18.6)$ & $74(81.4)$ & \\
\hline Diffuse & $13(50.0)$ & $13(50.0)$ & \\
\hline Recurrence & & & .120 \\
\hline \multicolumn{4}{|l|}{ Present } \\
\hline Local recurrence & $2(15.4)$ & $2(15.4)$ & \\
\hline Distant metastasis & $6(46.2)$ & $3(23.0)$ & \\
\hline Absent & $22(21.2)$ & $82(78.8)$ & \\
\hline Lymphovascular invasion & & & .015 \\
\hline Present & $18(36.0)$ & $32(64.0)$ & \\
\hline Absent & $12(17.9)$ & $55(82.1)$ & \\
\hline Perineural invasion & & & .004 \\
\hline Present & $13(46.4)$ & 15 (53.6) & \\
\hline Absent & $17(19.1)$ & 72 (80.9) & \\
\hline
\end{tabular}

Values are presented as number (\%).

EGC, early gastric cancer; AGC, advanced gastric cancer. 
mersed in $10 \mathrm{mmol} / \mathrm{L}$ citrate buffer $[\mathrm{pH} 6.0]$ and microwaved for 25 minutes). Slides were stained using an automatic staining device: Bond-X autoimmunostainer (Leica Microsystems, Wetzlar, Germany) with Bond Polymer Refine Detection System (Leica Microsystems). The following monoclonal mouse antibodies were used: anti-calretinin (1:50, Novocastra, Newcastle upon Tyne, UK), anti-WT1 (1:100, DAKO, Carpinteria, CA, USA), and anti-mesothelin (1:20; Leica). All slides were subsequently counterstained with hematoxylin. The mesothelial hyperplasia of pleura was used as a positive control. For a negative control, nonimmune mouse serum was used as a substitute for primary antibodies.

Membranous staining for mesothelin, nuclear and cytoplasmic staining for calretinin, and nuclear staining for WT1 were considered positive. We categorized any tumor with $\geq 5 \%$ staining of any intensity as positive and $<5 \%$ as negative. ${ }^{12}$ Stained sections were evaluated independently by two observers.

\section{Statistical analysis}

Statistical analysis was performed using SPSS ver. 21.0 (IBM Corp., Armonk, NY, USA). Correlations between the expression of mesothelial markers in gastric adenocarcinoma and clinicopathological variables were assessed using Pearson's $\chi^{2}$-test. The Kaplan-Meier method was used for DFS analysis, and differences were determined using log-rank test. The prognostic implications of mesothelin expression and clinicopathological parameters were analyzed by Cox univariate and multivariate proportional hazards models. DFS was calculated from the date of surgery to the time of first local recurrence or distant metastasis. Values of $\mathrm{p}<.05$ were considered statistically significant.
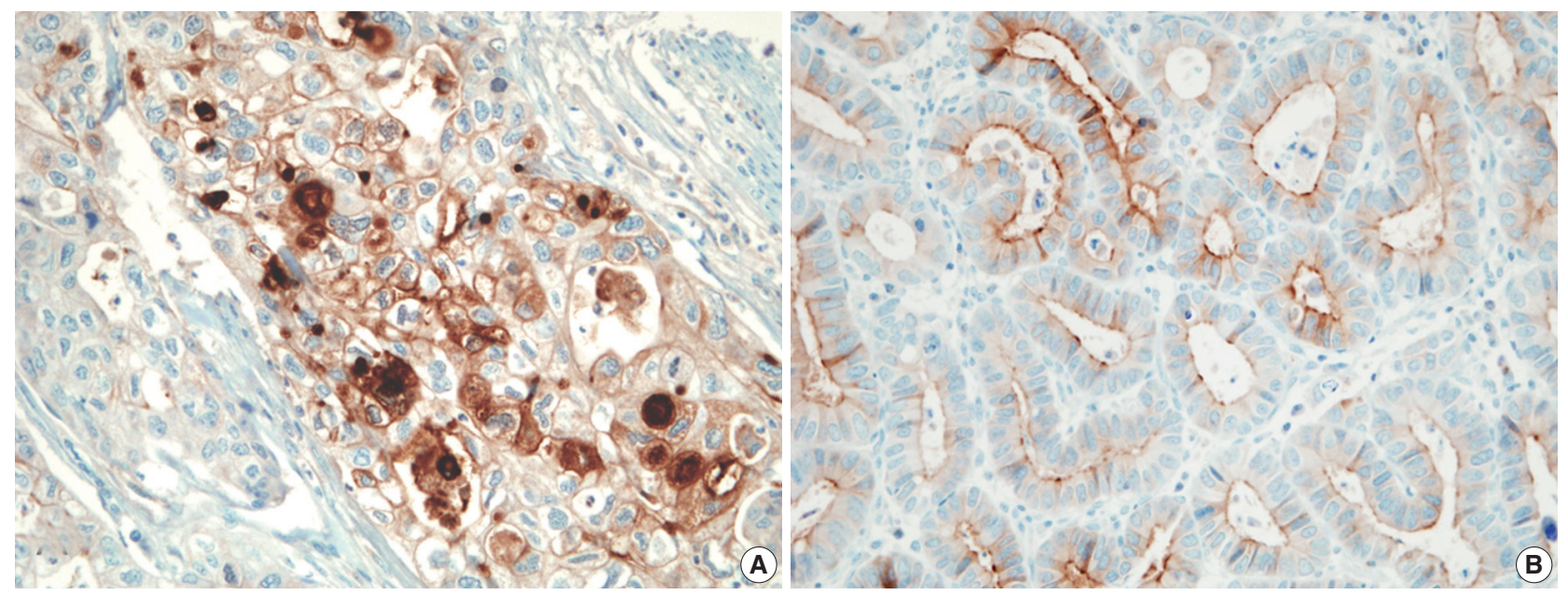

Fig. 1. The tumor cell membrane was stained with mesothelin in gastric adenocarcinomas, showing membranous (A) or apical patterns (B).
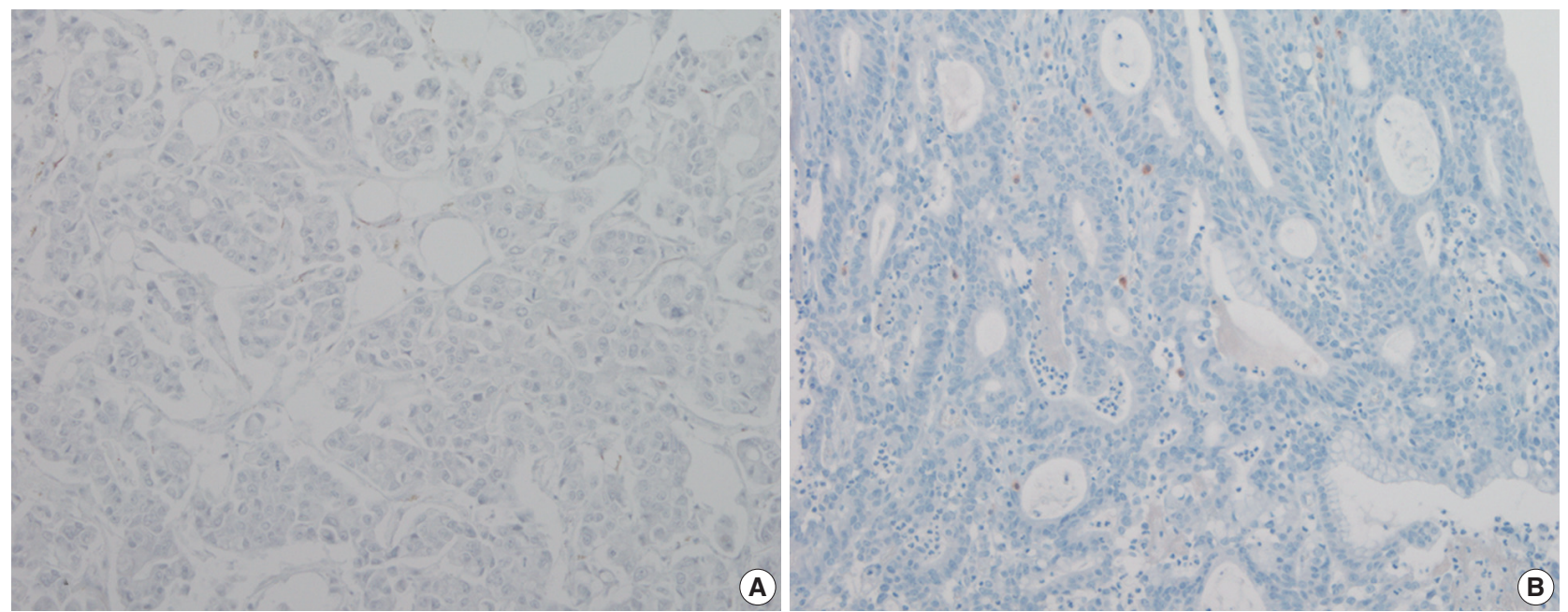

Fig. 2. Wilms tumor protein $1(A)$ and calretinin $(B)$ are not expressed in gastric adenocarcinoma. 


\section{RESULTS}

\section{Mesothelin, calretinin, and WT1 expression in gastric adenocarcinoma}

The pattern of mesothelin expression was characterized by a diffuse and strong pattern with thick, membranous staining, especially along the apical cell membrane (Fig. 1). Some tumor cells showed cytoplasmic staining. Mesothelin was positively stained in 30 cases (25.6\%). Staining only along the apical cell membrane was observed in 20 cases. In the remaining 10 cases, the staining was mixed membranous and cytoplasmic. Twentytwo cases exhibited cytoplasmic staining, which were considered negative. None of the 117 gastric adenocarcinomas (100\%) stained for calretinin or WT1 (Fig. 2). In normal gastric mucosa, mesothelin, calretinin, and WT1 were not expressed.

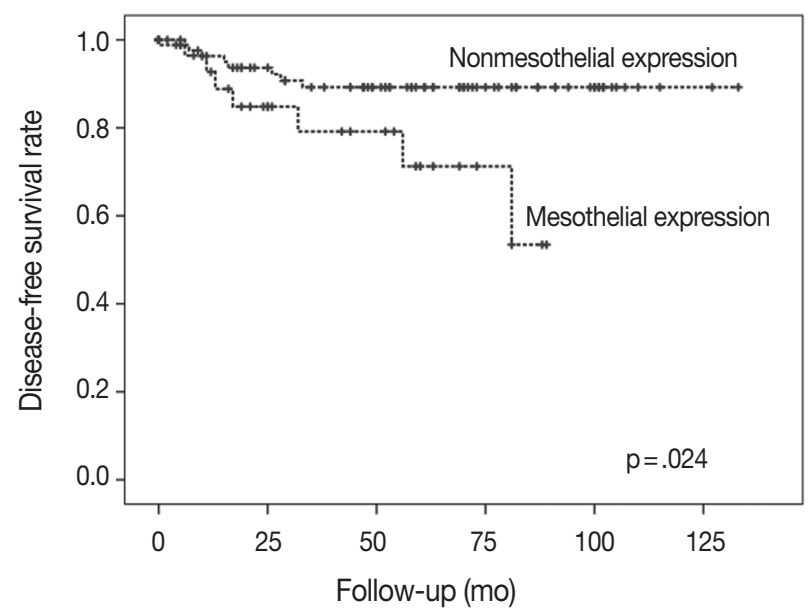

Fig. 3. The group with nonmesothelin expression had significantly better survival than the group with mesothelin expression.

\section{Association of mesothelin expression with clinicopathological variables}

The associations between mesothelin expression and clinicopathological characteristics in gastric adenocarcinoma are shown in Table 1. Mesothelin expression was related to increased depth of invasion ( $\mathrm{p}=.002)$, lymph node metastasis $(\mathrm{p}=.013)$, presence of lymphovascular invasion ( $\mathrm{p}=.015)$, and presence of perineural invasion ( $\mathrm{p}=.004)$.

\section{Mesothelin expression and DFS}

There was a difference in DFS between patients with mesothelin expression and patients with no mesothelin expression (median, 29 months vs 57 months). Patients with mesothelin expression had significant shorter DFS rate compared with those with no mesothelin expression ( $\mathrm{p}=.024$ ) (Fig. 3).

Cox proportional hazards regression model was used to evaluate the association between mesothelin expression and DFS. On univariate analysis, factors associated with DFS included mesothelial expression, depth of invasion, and perineural invasion (Table 2). However, multivariate Cox regression analysis suggested that mesothelin expression was not an independent prognostic factor (Table 2).

\section{DISCUSSION}

In this study, we obtained clinicopathological data related to expression of mesothelin, one of the three mesothelial markers. Approximately $25.6 \%$ of gastric adenocarcinomas showed positive staining for mesothelin. Importantly, we found that mesothelin expression was related to advanced disease and DFS. Therefore, mesothelin might be a promising therapeutic target in patients with advanced disease.

Mesothelin is a $40-\mathrm{kDa}$ cell surface glycoprotein. ${ }^{17}$ Its gene

Table 2. Univariate and multivariate analyses for disease-free survival

\begin{tabular}{|c|c|c|c|c|c|c|c|}
\hline \multirow{2}{*}{ Variable } & & \multicolumn{3}{|c|}{ Univariate } & \multicolumn{3}{|c|}{ Multivariate } \\
\hline & & $\mathrm{HR}$ & $95 \% \mathrm{Cl}$ & $p$-value & $\mathrm{HR}$ & $95 \% \mathrm{Cl}$ & $p$-value \\
\hline \multirow[t]{2}{*}{ Stage } & EGC & 1 & $1.152-5.321$ & .001 & 1 & $2.036-9.922$ & .004 \\
\hline & AGC & 3.240 & & & 3.521 & & \\
\hline \multirow[t]{2}{*}{ Lymphovascular invasion } & Absent & 1 & $0.572-2.801$ & .075 & - & - & - \\
\hline & Present & 1.283 & & & - & & \\
\hline \multirow[t]{2}{*}{ Lymph node metastasis } & Absent & 1 & $0.961-5.027$ & .058 & - & - & - \\
\hline & Present & 1.502 & & & - & & \\
\hline \multirow[t]{2}{*}{ Perineural invasion } & Absent & 1 & $1.254-6.455$ & .001 & 1 & $0.356-9.616$ & .475 \\
\hline & Present & 2.428 & & & 1.847 & & \\
\hline \multirow[t]{2}{*}{ Mesothelin expression } & Negative & 1 & $1.523-5.212$ & .019 & 1 & $0.251-2.703$ & .770 \\
\hline & Positive & 2.261 & & & 1.428 & & \\
\hline
\end{tabular}

HR, hazard ratio; 95\% Cl, 95\% confidence interval; EGC, early gastric cancer; AGC, advanced gastric cancer. 
encodes a precursor protein that is cleaved by proteases to form $40-\mathrm{kDa}$ mesothelin that attaches to the cell membrane and a $31-\mathrm{kDa}$ cleaved fragment, which is secreted into the blood. Mesothelin is believed to be involved in cell adhesion, though its biological function is not well known. Einama et al. ${ }^{11}$ have investigated mesothelin expression in gastric adenocarcinoma, focusing on localization of mesothelin expression. They found that luminal membranous expression was a significant prognostic factor in gastric cancer, while cytoplasmic expression was not significantly correlated with prognosis of gastric cancer. ${ }^{13}$ In their study, membranous or mixed membranous and cytoplasmic expression was considered positive staining. In addition to mesothelin expression pattern, previous studies have used various scoring systems to quantify immunohistochemically observed expression of mesothelin in different tumor types. ${ }^{9-12,18}$ Because the staining patterns of mesothelin were quite distinct, such as diffuse and strong with thick, membranous staining or no staining, we considered $\geq 5 \%$ staining of any intensity as positive, using the cut-off described by Baba et al. ${ }^{12}$ Thus, the rate of mesothelin expression in gastric adenocarcinomas was $25.6 \%$ in our study. However, the rate of mesothelin expression differed between studies. Taliano et al. ${ }^{14}$ reported that mesothelin was expressed in $59 \%$ of gastric adenocarcinomas. Lugli et al. ${ }^{13}$ found that mesothelin expression was $15.4 \%$ in the membrane. These differences between our results and prior studies may be attributed to the different sample sizes and distribution as well as different staining protocols and antibodies used.

Mesothelin is expressed in several cancers, including epithelioid mesotheliomas, pancreatic and biliary adenocarcinomas, and ovarian and gastric cancers. ${ }^{11,12,18-20}$ The relationships between mesothelin expression and tumor aggressiveness and clinical outcomes have been studied in various cancers. The results of this study confirmed that mesothelin expression was strongly associated with depth of invasion, lymph node metastasis, and presence of lymphovascular and perineural invasion. Similarly, Baba et al. ${ }^{12}$ reported that mesothelin expression was correlated with greater nodal involvement and deeper invasion. Einama et $a l{ }^{11}$ also reported that mesothelin expression was associated with clinical stage, lymphatic and blood vessel permeation, and recurrence. However, previous studies have reported conflicting results regarding the relationship between mesothelin expression and clinical outcome. Kawamata et al. ${ }^{9}$ reported that a mesothelin-positive group showed poor prognosis in several gastrointestinal malignancies, including biliary adenocarcinoma. In contrast, Yen et al..$^{10}$ demonstrated that mesothelin expression was associated with prolonged survival in patients with advanced stage epithelial ovarian carcinomas. For patients with gastric cancer, studies of the association between mesothelin expression and survival have shown different results. Baba et al. ${ }^{12}$ found that mesothelin expression was correlated with prolonged patient survival in AGC. In addition to our study, a previous study has reported that mesothelin expression was correlated with poor overall survival. ${ }^{11}$

Although the mechanism by which mesothelin affects survival of patients with gastric cancer remains unclear, discrepancies between studies may be related to the biological role of mesothelin. Recent studies have reported that mesothelin plays a role in tumorigenesis by increasing cellular proliferation and migration. Servais et al. ${ }^{21}$ found that mesothelin promoted tumor cell invasion by increasing matrix metalloprotease-9 secretion in mesothelioma. Additionally, Rump et al. ${ }^{22}$ observed that mesothelin bound to carbohydrate antigen 125/MUC16 with very high affinity and may contribute to the adhesion of tumor cells in peritoneal metastasis. Bharadwaj et al. ${ }^{23}$ reported that mesothelin expression increased resistance to tumor necrosis factor- $\alpha-$ induced apoptosis through Akt/phosphoinositide 3-kinase/nuclear factor $\mathrm{\kappa B}$ activation. Mesothelin-overexpressing pancreatic cancer cell lines showed increased cyclin $\mathrm{E}$ and cyclin-dependent kinase 2 expression, resulting in increased cell proliferation and cell cycle progression. ${ }^{24}$ These findings suggest significant interplay between mesothelin-related proteins and/or genes and aggressive tumor behavior.

Increasing evidence has suggested that mesothelin is a strongly immunogenic protein. Thomas et al. ${ }^{25}$ reported that pancreatic cancer patients who were vaccinated with granulocyte macrophage colony-stimulating factor-secreting pancreatic tumor cells showed a strong mesothelin-specific CD8 + T-cell immune response. Ho et al. ${ }^{26}$ detected mesothelin-specific IgG antibodies in the serum of patients with advanced mesothelioma and ovarian cancer. These results indicate that mesothelin-specific B-cell and T-cell responses against mesothelin-expressing carcinoma cells contribute to overall prolonged survival. The reason for differences in clinical outcomes among various carcinomas remains unclear, and additional studies are necessary to explain these discrepancies.

Both silencing of mesothelin for inhibited cell proliferation and migration and increased mesothelin-specific immune response may be correlated with the control of tumor progression. Thus, mesothelin has been evaluated as a marker for biological malignancy from both genetic and immunological perspectives. Mesothelin is a promising target for immune-based therapy, specifically for mesothelioma and pancreatic and ovarian cancers. 
Several ongoing clinical trials in patients with these carcinomas have revealed a beneficial effect. ${ }^{27,28}$ For example, SS1P is a representative recombinant antimesothelin immunotoxin, and the therapy response rate of SS1P combined with chemotherapy exhibits good response. ${ }^{28}$

We hypothesized that calretinin and WT1 would show positive reactivity in gastric adenocarcinoma. ${ }^{13-15}$ No prior studies have evaluated WT1 expression in gastric adenocarcinoma. A few studies have reported calretinin expression in a wide variety of poorly differentiated carcinomas, including gastric adenocarcinoma. ${ }^{13}$ In this study, we found that neither calretinin nor WT1 was expressed. This discrepancy from previous study ${ }^{13}$ is likely related to technique sensitivity, resulting in false-positive values, and may also be related to the diversity of antibodies used.

Given the conflicting results from previous investigations, we evaluated the expression pattern and prognostic value of mesothelin in gastric adenocarcinoma and the correlations of mesothelin expression with clinicopathological variables. We found that $25.6 \%$ of gastric adenocarcinomas expressed mesothelin, which was associated with poor prognosis. Our results suggest that mesothelin-targeted therapies, such as SS1P, are important alternatives for cancer patients with mesothelin expression.

\section{Conflicts of Interest}

No potential conflict of interest relevant to this article was reported.

\section{Acknowledgments}

This work was supported by a grant from Inje University in 2009.

\section{REFERENCES}

1. Ferlay J, Soerjomataram I, Dikshit R, et al. Cancer incidence and mortality worldwide: sources, methods and major patterns in GLOBOCAN 2012. Int J Cancer 2015; 136: E359-86.

2. Oh CM, Won YJ, Jung KW, et al. Cancer statistics in Korea: incidence, mortality, survival, and prevalence in 2013. Cancer Res Treat 2016; 48: 436-50.

3. Gotoda T. Endoscopic resection of early gastric cancer. Gastric Cancer 2007; 10: 1-11.

4. Oba K, Paoletti X, Bang YJ, et al. Role of chemotherapy for advanced/recurrent gastric cancer: an individual-patient-data meta- analysis. Eur J Cancer 2013; 49: 1565-77.

5. Wagner AD, Grothe W, Haerting J, Kleber G, Grothey A, Fleig WE. Chemotherapy in advanced gastric cancer: a systematic review and meta-analysis based on aggregate data. J Clin Oncol 2006; 24: 2903-9.

6. Kataoka H, Mori Y, Shimura T, et al. A phase II prospective study of the trastuzumab combined with 5-weekly S-1 and CDDP therapy for HER2-positive advanced gastric cancer. Cancer Chemother Pharmacol 2016; 77: 957-62.

7. Boku N. HER2-positive gastric cancer. Gastric Cancer 2014; 17: 1-12.

8. Ordóñez NG. Value of mesothelin immunostaining in the diagnosis of mesothelioma. Mod Pathol 2003; 16: 192-7.

9. Kawamata F, Kamachi H, Einama T, et al. Intracellular localization of mesothelin predicts patient prognosis of extrahepatic bile duct cancer. Int J Oncol 2012; 41: 2109-18.

10. Yen MJ, Hsu CY, Mao TL, et al. Diffuse mesothelin expression correlates with prolonged patient survival in ovarian serous carcinoma. Clin Cancer Res 2006; 12(3 Pt 1): 827-31.

11. Einama T, Homma S, Kamachi H, et al. Luminal membrane expression of mesothelin is a prominent poor prognostic factor for gastric cancer. Br J Cancer 2012; 107: 137-42.

12. Baba K, Ishigami S, Arigami T, et al. Mesothelin expression correlates with prolonged patient survival in gastric cancer. J Surg Oncol 2012; 105: 195-9.

13. Lugli A, Forster $Y$, Haas $P$, et al. Calretinin expression in human normal and neoplastic tissues: a tissue microarray analysis on 5233 tissue samples. Hum Pathol 2003; 34: 994-1000.

14. Taliano RJ, Lu S, Singh K, et al. Calretinin expression in high-grade invasive ductal carcinoma of the breast is associated with basal-like subtype and unfavorable prognosis. Hum Pathol 2013; 44: 2743-50.

15. Kao SC, Klebe S, Henderson DW, et al. Low calretinin expression and high neutrophil-to-lymphocyte ratio are poor prognostic factors in patients with malignant mesothelioma undergoing extrapleural pneumonectomy. J Thorac Oncol 2011; 6: 1923-9.

16. Qi XW, Zhang F, Wu H, et al. Wilms' tumor 1 (WT1) expression and prognosis in solid cancer patients: a systematic review and meta-analysis. Sci Rep 2015; 5: 8924.

17. Chang K, Pastan I. Molecular cloning of mesothelin, a differentiation antigen present on mesothelium, mesotheliomas, and ovarian cancers. Proc Natl Acad Sci U S A 1996; 93: 136-40.

18. Nomura R, Fujii H, Abe M, et al. Mesothelin expression is a prognostic factor in cholangiocellular carcinoma. Int Surg 2013; 98: 164-9.

19. Argani P, Iacobuzio-Donahue C, Ryu B, et al. Mesothelin is overexpressed in the vast majority of ductal adenocarcinomas of the pancreas: identification of a new pancreatic cancer marker by serial 
analysis of gene expression (SAGE). Clin Cancer Res 2001; 7: 3862-8.

20. Hassan R, Kreitman RJ, Pastan I, Willingham MC. Localization of mesothelin in epithelial ovarian cancer. Appl Immunohistochem Mol Morphol 2005; 13: 243-7.

21. Servais EL, Colovos C, Rodriguez L, et al. Mesothelin overexpression promotes mesothelioma cell invasion and MMP-9 secretion in an orthotopic mouse model and in epithelioid pleural mesothelioma patients. Clin Cancer Res 2012; 18: 2478-89.

22. Rump A, Morikawa Y, Tanaka M, et al. Binding of ovarian cancer antigen CA125/MUC16 to mesothelin mediates cell adhesion. J Biol Chem 2004; 279: 9190-8.

23. Bharadwaj U, Marin-Muller C, Li M, Chen C, Yao Q. Mesothelin confers pancreatic cancer cell resistance to TNF-alpha-induced apoptosis through Akt/PI3K/NF-kappaB activation and IL-6/ Mcl-1 overexpression. Mol Cancer 2011; 10: 106.

24. Bharadwaj U, Li M, Chen C, Yao Q. Mesothelin-induced pancreatic cancer cell proliferation involves alteration of cyclin E via activa- tion of signal transducer and activator of transcription protein 3. Mol Cancer Res 2008; 6: 1755-65.

25. Thomas AM, Santarsiero LM, Lutz ER, et al. Mesothelin-specific CD8(+) T cell responses provide evidence of in vivo cross-priming by antigen-presenting cells in vaccinated pancreatic cancer patients. J Exp Med 2004; 200: 297-306.

26. Ho M, Hassan R, Zhang J, et al. Humoral immune response to mesothelin in mesothelioma and ovarian cancer patients. Clin Cancer Res 2005; 11: 3814-20.

27. Hassan R, Ho M. Mesothelin targeted cancer immunotherapy. Eur J Cancer 2008; 44: 46-53.

28. Hassan R, Sharon E, Thomas A, et al. Phase 1 study of the antimesothelin immunotoxin SS1P in combination with pemetrexed and cisplatin for front-line therapy of pleural mesothelioma and correlation of tumor response with serum mesothelin, megakaryocyte potentiating factor, and cancer antigen 125 . Cancer 2014; 120: 3311-9. 